\title{
Exploring Neutral Hydrogen and Galaxy Evolution with the SKA
}

S. -L. Blyth ${ }^{1}$, J. M. van der Hulst ${ }^{* 2}$, M.A.W. Verheijen ${ }^{2}$, HI SWG Members ${ }^{3}$, B. Catinella $^{4}$, F. Fraternali ${ }^{5,2}$, M. P. Haynes ${ }^{6}$, K. M. Hess ${ }^{1,2,7}$, B.S. Koribalski ${ }^{8}$, C. Lagos $^{9}$, M. Meyer ${ }^{10}$, D. Obreschkow ${ }^{10}$, A. Popping ${ }^{10,11}$, C. Power ${ }^{10}$, L. Verdes-Montenegro ${ }^{12}$, and M. Zwaan ${ }^{9}$

${ }^{1}$ University of Cape Town, South Africa; ${ }^{2}$ Kapteyn Astronomical Institute, The Netherlands;

${ }^{3}$ SKA HI Science Working Group; ${ }^{4}$ Swinburne University of Technology, Australia; ${ }^{5}$ University of Bologna, Italy; ${ }^{6}$ Cornell University, USA; ${ }^{7}$ Netherlands Institute for Radio Astronomy (ASTRON); ${ }^{8}$ ATNF, CSIRO Astronomy and Space Science, Australia; ${ }^{9}$ European Southern Observatory, Germany; ${ }^{10}$ ICRAR, University of Western Australia; ${ }^{11}$ ARC Centre of Excellence for All-sky Astrophysics (CAASTRO); ${ }^{12}$ Instituto de Astrofisica de Andalucia (IAA/CSIC), Spain E-mail: sarblytheast.uct.ac.za

One of the key science drivers for the development of the SKA is to observe the neutral hydrogen, $\mathrm{HI}$, in galaxies as a means to probe galaxy evolution across a range of environments over cosmic time. Over the past decade, much progress has been made in theoretical simulations and observations of $\mathrm{HI}$ in galaxies. However, recent $\mathrm{H}$ I surveys on both single dish radio telescopes and interferometers, while providing detailed information on global H I properties, the dark matter distribution in galaxies, as well as insight into the relationship between star formation and the interstellar medium, have been limited to the local universe. Ongoing and upcoming H I surveys on SKA pathfinder instruments will extend these measurements beyond the local universe to intermediate redshifts. We present here an overview of the $\mathrm{H}$ I science which will be possible with the the SKA and which will build upon the expected increase in knowledge of $\mathrm{HI}$ in and around galaxies obtained with the SKA pathfinder surveys. With the SKA1 the greatest improvement is the capability to image galaxies at reasonable linear resolution and good column density sensitivity to much higer redshifts $(0.2<z<1.7)$. So one will not only be able to increase the number of detections to study the evolution of the $\mathrm{H}$ I mass function, but also have the sensitivity and resolution to study inflows and outflows to and from galaxies and the kinematics of the gas within and around galaxies as a function of environment and cosmic time out to previously unexplored depths. The increased sensitivity of SKA2 will allow us to image Milky Way-size galaxies out to redshifts of $z=1$ and will provide the data required for a comprehensive picture of the $\mathrm{HI}$ content of galaxies back to $z \sim 2$ when the cosmic star formation rate density was at its peak.

Advancing Astrophysics with the Square Kilometre Array

June 8-13, 2014

Giardini Naxos, Italy

${ }^{*}$ Speaker 


\section{Introduction}

How galaxies form and evolve is one of the fundamental questions in modern astrophysics. Over the past decade, multiwavelength observations of galaxies spanning a wide range of cosmic time have indicated that galaxy evolution seems to depend primarily on two parameters: a galaxy's stellar mass, and the environment in which it is located. In the $\Lambda$-cold dark matter (CDM) hierarchical structure formation picture, galaxies form through the successive mergers of smaller units (namely dark matter haloes) to form larger structures. Prior to the epoch of galaxy formation, baryons existed almost entirely in gaseous form, and it is through the infall of this material onto the filamentary structures of the cosmic web, its accretion into the deepest potential wells, and ultimate collapse into dense molecular clouds, that galaxies were able to start forming and producing stars. The ongoing influence of dark matter haloes, and complex feedback mechanisms between baryonic components, continue to regulate the gas content and evolution of galaxies today. In order to fully understand the build up of stellar mass in galaxies, we need to understand the role that neutral gas, the fuel for star formation, plays, along with the physical processes involved. Therefore we need to study the neutral gas distributions and kinematics of galaxies, in large numbers, in different environments, as a function of cosmic time to uncover the full picture of galaxy evolution and structure formation in the Universe. This is one of the main scientific drivers for the development of the SKA.

Along with recent progress in observations, there have been many theoretical advances in both semi-analytic modelling and full hydrodynamical modelling of galaxy evolution and, importantly, the role of neutral hydrogen gas, in both its atomic (H I) and molecular forms (see section 2.2). However, observations of neutral gas have lagged behind observations at other wavelengths; due to the intrinsic faintness of $\mathrm{HI}$ emission, unreasonably long observing times are required to probe to redshifts beyond $z \sim 0.25$ with existing facilities, although with the construction of SKA pathfinder instruments and significant upgrades to current facilities, deeper, more sensitive, observations will be possible in the very near future (see section 2.3). The SKA will allow us to probe HI emission in galaxies to previously unexplored depths and further back in cosmic history. The SKA2 will enable optical quality H I imaging of Milky Way size galaxies out to redshifts of $z=1$

Analysis of thousands of galaxies in the Sloan Digital Sky Survey (SDSS) has shown that they follow a bimodal distribution in colour as a function of stellar mass (Baldry et al. (2004)); most galaxies are either located in the so-called blue cloud or on the red sequence, indicating that star formation is either still ongoing or was quenched billions of years ago. Furthermore, the galaxy 'main sequence' (star formation rate vs. stellar mass) shows a relatively smooth evolution over cosmic time from $z \sim 2.5$ to $z \sim 0$ while over the same period, the star formation rate density of the Universe has dropped by more than an order of magnitude since its peak at $z \sim 2-3$ (Madau et al. (1998); Hopkins \& Beacom (2006)). Star formation also seems to have shifted from the more massive galaxies to less massive galaxies at later times, a pattern known as cosmic downsizing (Cowie et al. (1996)). Understanding the role of the neutral gas that fuels star formation and enables the build up of stellar mass as well as the role of the environment in enabling or quenching these processes, is vital to disentangle the physics involved in the evolution of galaxies. To move forward, a number of key questions related to the role of $\mathrm{H} \mathrm{I}$ in galaxies need to be answered such as:

- What is the distribution and kinematics of the neutral (H I ) gas within and around galaxies, 
both as a function of environment (i.e. groups/clusters vs. the field) and over cosmic time?

- How much $\mathrm{H}$ I is there on average as a function of redshift?

- How does the $M_{H I}$ of galaxies scale with their stellar/halo masses and other properties, e. g.,star formation rate, as a function of environment and redshift?

- How important is gas accretion vs. merging in terms of building stellar mass?

The process of star formation is intimately related to how individual galaxies evolve. On kpc-size scales, the Kennicutt-Schmidt law (Bigiel et al. (2008); Leroy et al. (2012); Calzetti et al. (2012)) describes the relationship between the star formation rate surface density and the molecular gas mass surface density. However, details of the conditions needed for star formation to begin at the scales of (molecular) clouds are as yet unclear. High resolution observations of the interstellar medium (ISM) in individual galaxies are required in order to clarify the astrophysical processes taking place at these scales. This topic will be more fully discussed in a separate chapter, The Interstellar medium in Galaxies (de Blok et al. (2014)). Further discussion of the interaction of galaxies with their environment, the IGM and the distribution of neutral hydrogen in the cosmic web will be presented in the chapter The Intergalactic Medium and the Cosmic Web (Popping et al. (2014)).

Observations of $\mathrm{HI}$ in absorption along lines of sight to strong radio sources can provide information on the neutral gas content of galaxies at higher redshifts than it is possible to reach directly with $\mathrm{HI}$ emission measurements. These are key measurements for studies of galaxy evolution at higher redshifts, and will be discussed further in a separate chapter, Cool Outflows and HI absorbers (Morganti et al. (2014))

\section{H I observations and theory: the status quo}

\subsection{Recent observational results}

\subsubsection{The Local Universe}

Recent years have ushered in a "golden age" of $\mathrm{HI}$ science that has seen a variety of $\mathrm{H} \mathrm{I}$ surveys to study galaxy evolution take place. The recent single dish, large area blind $\mathrm{H}$ I surveys HIPASS (Barnes et al. (2001)) and ALFALFA (Giovanelli et al. (2005)) have shed light on the global H I properties of galaxies in general and provided the best estimates of the global H I mass function. Poor statistics at the very high $\mathrm{H}_{\mathrm{I}}$ mass end and the very low $\mathrm{H}$ I mass end $\left(<10^{7}\right.$ $\mathrm{M}_{\odot}$ ) limit the current results and do not resolve questions such as whether the $\mathrm{H}$ I mass function depends on the local enviroment (Zwaan et al. (2005); Springob et al. (2005)). Yet these surveys have revealed that there is indeed an extreme paucity of $\mathrm{HI}$ objects without stellar counterparts when the HI mass detection limit is pushed well below $5 \times 10^{6} \mathrm{M}_{\odot}$, enabling the interesting discovery of a few local, gas-rich dwarf galaxies such as Leo T (Ryan-Weber et al. (2008)) and Leo $\mathrm{P}$ (Giovanelli et al. (2013)). In addition the surveys demonstrate the power of combining the H I survey information with data from other wavebands (optical, UV, IR) to address questions relevant to galaxy evolution (Catinella et al. (2010); Schiminovich et al. (2010); Wong et al. (2009)).

More detailed information about the kinematics and structure of the H I disks in individual galaxies has come from resolved observations using synthesis radio telescopes. Major surveys 
carried out in the last decade are WHISP (van der Hulst et al. (2001)), THINGS (Walter et al. (2008)), LVHIS (Koribalski (2008)), Atlas-3D (Serra et al. (2012)), Little THINGS (Hunter et al. (2012)), VLA-ANGST (Ott et al. (2012)), VIVA (Chung et al. (2009)), SHIELD (Cannon et al. (2011)), FIGGS (Begum et al. (2008)), HALOGAS (Heald et al. (2011)) and BlueDisks (Wang et al. (2013)). Major results are the systematic inventory of the distribution of dark matter in galaxies, the prevalence of warps, tidal features, and signs of ram pressure stripping, and detailed relationships between star formation and the ISM including galactic fountains and the presence of extra-planar H I . A synopsis of results to date can be found in two recent reviews (Sancisi et al. (2008); van der Hulst \& de Blok (2013)).

Observations of $\mathrm{HI}$ in galaxies in very dense environments (a well studied example is the Virgo cluster (Chung et al. (2009)) demonstrate the importance of the environment. The growing inventory of detailed H I observations of galaxies in different environments is mixed, but suggest that even intermediate density environments in galaxy groups (Hess \& Wilcots (2013)), and on the outskirts of clusters (Jaffé et al. (2013)) impact the HI content of galaxies. Dense environments in small volumes, such as Hickson compact groups, also exhibit H I deficiencies (Verdes-Montenegro et al. (2001)) with a suggestion that a reservoir of low density H I might exist to feed the galaxies (Borthakur et al. (2011)). In very isolated environments, such as voids (Kreckel et al. (2012); Beygu et al. (2013)) there is a suggestion that accretion is an important process. Obtaining information for galaxies that are specifically selected to be extremely isolated will be very important (Espada et al. (2011)) provided detailed imaging is performed (Scott et al. (2014)). Having a good enough sample of $\mathrm{HI}$ in and around many galaxies in different enviroments to address the question of nature versus nurture will will be a prime goal for the SKA.

Three surveys are currently pushing the present technology to redshifts beyond $z=0.2$; the HIGHz survey (Catinella et al. (2008)) with Arecibo and two interferometric surveys, BUDHIES (Verheijen et al. (2007); Jaffé et al. (2013)) and CHILES (Fernández et al. (2013)) which are using observing times approaching 1000 hours. To date, the most successful method of detecting $\mathrm{H} \mathrm{I}$ emission beyond $z=0.2$ with current instrumentation is stacking based on position and redshift information from optical surveys. In the following sections we will briefly describe the current status of $\mathrm{H}$ I observations and then focus on the important next steps to be addressed by SKA1 and SKA2.

\subsubsection{Observations at low redshift $(z<0.4)$}

Exploiting the exquisite sensitivity of the Arecibo radio telescope, the $\mathrm{HIGHz}$ survey provides a glimpse into the $\mathrm{H}$ I properties of star-forming galaxies at $z \sim 0.2$ located in relatively isolated environments. The survey measured the $\mathrm{H}$ I content of 39 optically-selected galaxies with redshifts between $0.17<z<0.25$; these are all actively star-forming, disk-dominated systems with stellar and $\mathrm{HI}$ masses larger than $10^{10} \mathrm{M}_{\odot}$. This sample includes not only the highest-redshift detections of $\mathrm{H}$ I emission from individual galaxies to date, but also some of the most $\mathrm{H} \mathrm{I}$-massive systems known. Despite being exceptionally large, the H I reservoirs of these galaxies are consistent with what is expected from their UV and optical properties. This, and the fact that the galaxies lie on the baryonic Tully-Fisher relation, suggests that HIGHz systems are rare, scaled-up versions of gasrich disks in the local Universe. These observations provide important insight into the properties 
of the massive, H I-rich systems that will likely dominate the next-generation H I surveys with the SKA and its pathfinder telescopes.

Taking advantage of the upgraded Westerbork receiver system, a Blind, Ultra-Deep H I Environmental Survey (BUDHIES) has been carried out to image two cosmic volumes, both covering the redshift range $\mathrm{z}=0.164-0.224$, centered on the galaxy clusters Abell 2192 at $z=0.187$ and Abell 963 at $z=0.206$. A963 is a massive, lensing, X-ray bright, Butcher-Oemler cluster with an unusual high fraction of blue galaxies in its center. The main scientific goal of BUDHIES is to characterize the gas content of these blue galaxies in relation to the gas content of galaxies in the cluster outskirts and the surrounding field in which this cluster is embedded. A 2192 is a less massive cluster that mainly serves as a control object. The clusters within their Abell radii occupy only $\sim 5 \%$ of the total surveyed volume, which also contains voids and galaxy overdensities in the fore- and background of both clusters.

Data were collected with the WSRT with $78 \times 12^{\mathrm{hr}}$ on A2192 and $117 \times 12^{\mathrm{hr}}$ on A963 to obtain a similar $\mathrm{HI}$ mass limit of $2 \times 10^{9} \mathrm{M}_{\odot}$. A total of 39 galaxies were detected in the volume containing A2192 and 120 galaxies in A963. First results from a pilot survey showed that none of the blue galaxies in A963 were detected, while stacking the HI spectra from galaxies of similar luminosity and color in the surrounding field showed a clear detection (Verheijen et al. (2007)). The full dataset is currently being analysed with a focus on the $\mathrm{H}$ I content of galaxies in the various substructures within and around the clusters (Jaffé et al. (2012, 2013)), as well as the H I-based Tully-Fisher relation at these redshifts.

The COSMOS H i Large Extragalactic Survey (CHILES) is an ongoing 1000 hour program designed to take advantage of the upgraded capabilities on the Karl G. Jansky Very Large Array (VLA). The upgrade enables continuous redshift coverage for $\mathrm{H}$ I in emission from $z=0-0.5$, doubling the look-back time of existing $\mathrm{H}$ I measurements. A single pointing centered in the COSMOS field, CHILES is expected to detect and resolve Milky Way-like galaxies in H I $\left(M_{H I}=\right.$ $3 \times 10^{10} \mathrm{M}_{\odot}$ ) at the most distant redshift accessible, and the most gas-rich galaxies that we know of at $z=0$, totalling over $300 \mathrm{HI}$-rich objects in the roughly $4 \times 10^{5} \mathrm{Mpc}^{3}$ surveyed. The chosen field intersects with known large-scale structure at low, intermediate, and high redshifts, and COSMOS provides a wealth of complementary multi-wavelength photometry and optical spectroscopy enabling the study of galaxy evolution as a function of galaxy properties, environment, and cosmic time. CHILES will be the first survey to probe the H I content, growth, and morphology of galaxies over the same redshift range in which star formation undergoes "cosmic downsizing".

First results from 50 hours observing (Fernández et al. (2013)) produced 33 direct detections in observations covering a continuous redshift range $z=0-0.193$ over a $34^{\prime} \times 34^{\prime}$ field of view, including three galaxies for which there were no previous spectroscopic redshifts. The most distant galaxy was detected at $\mathrm{z}=0.176$ and has an $\mathrm{HI}$ mass of $8 \times 10^{9} \mathrm{M}_{\odot}$. Stacking the H I spectra of 80 galaxies in a "wall" between $z=0.12-0.13$ produced a detection with an average HI mass of $1.8 \times 10^{9} \mathrm{M}_{\odot}$.

\section{Recent results from $\mathrm{H}$ I stacking}

H I observations have only begun to detect $21 \mathrm{~cm}$ line emission from individual galaxies beyond redshift $z \sim 0.1$. Although a number of galaxies will be individually detected at these and higher redshifts by the upcoming SKA pathfinder surveys (see Table 1), the most stringent constraints on 
the H I content of galaxies across cosmic time will come from stacking of undetected sources.

Stacking has become a common tool to constrain the statistical properties of a population of objects that lack individual detections in a survey, and has been applied to a variety of different astrophysical data, including $\mathrm{H}$ I -line spectra (Chengalur et al. (2001); Lah et al. (2007); Verheijen et al. (2007); Lah et al. (2009); Fabello et al. (2011); Delhaize et al. (2013); Geréb et al. (2013)). This technique requires independent measurements of the redshifts of the galaxies, and yields an estimate of the average $\mathrm{H}$ I content of a sample of galaxies by co-adding their line emission (see description in Fabello et al. (2011)).

Over the past decade, in an effort to probe the evolution of H I gas with cosmic time, Lah et al. (2007), who used the Giant Metrewave Radio Telescope (GMRT) to estimate the H I content of star-forming galaxies at $z \sim 0.24$, and Rhee et al. (2013), who targeted field galaxies at redshift $z \sim 0.1$ and $z \sim 0.2$ with the Westerbork Synthesis Radio Telescope (WSRT), showed that it is possible to constrain the cosmic H I gas density, $\Omega_{H I}$, of the Universe up to look-back times 2-4 Gyr with H I stacking. Intriguingly, these studies have begun to bridge the gap between high$\mathrm{z}$ damped Lyman- $\alpha$ observations and blind $21-\mathrm{cm}$ surveys at $z=0$ (Fig. 1). Determining $\Omega_{H I}$ is especially critical at these intermediate redshifts when the cosmic star formation rate density plummeted.

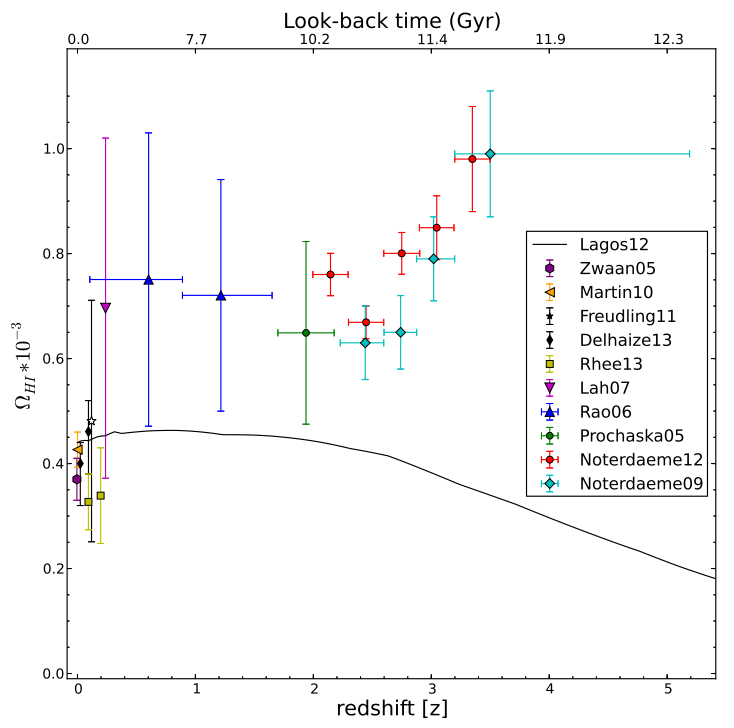

Figure 1: Recent compilation of cosmic $\mathrm{H} \mathrm{I}$ gas density measurements as functions of redshift and look-back time. At $\mathrm{z}=0$, the hexagon and triangle refer to direct observations from blind H I surveys (Zwaan et al. (2005); Martin et al. (2010)). All points above $z=0.4$ are damped Ly- $\alpha$ measurements from HST and SDSS (Prochaska et al. (2005); Rao et al. (2006); Noterdaeme et al. $(2009,2012))$. Bridging the gap between the two are the estimates from H I stacking of Parkes (diamonds)(Delhaize et al. (2013), WSRT (yellow squares) (Rhee et al. (2013)) and GMRT (magenta triangle) (Lah et al. (2007)) observations. The curve represents model predictions by Lagos et al. (2012).

Stacking is also extremely promising for galaxy evolution studies in general, as a means to investigate the connection between gas, star formation rate, and other galaxy properties across environment and time. Application of the stacking technique to a sample of $\sim 5000$ optically-selected galaxies with $\mathrm{H}$ I observations from ALFALFA has successfully recovered the main scaling relations connecting the gas fractions of galaxies with their structural and star formation properties (Fabello et al. (2011)) and the effects of environment on gas content (Fabello et al. (2012)). Because of the large statistics needed for these studies, and because of the need to reach the gas-poor regime, this question is optimally addressed by H I stacking, rather than by individually detected galaxies. Environmental effects on the gas have also been investigated at much greater distances, using $\mathrm{H} \mathrm{I}$ stacking at $z \sim 0.2$ (Verheijen et al. (2007)) and $z=0.37$ (Lah et al. (2009)). 
Clearly, the upcoming H I surveys with the SKA and its pathfinders will allow us to extend all these studies to significantly higher redshifts, and to vastly larger optically-selected samples than is possible today. $\mathrm{H}$ I stacking will be a very powerful technique to exploit the next-generation $\mathrm{H} \mathrm{I}$ surveys, well beyond the limits imposed by individual detections.

\subsection{Recent theoretical advances}

The two most widely used techniques to study galaxy formation in a cosmological context are hydrodynamical simulations and semi-analytical models (SAMs). Hydrodynamical simulations follow the evolution of both the dark matter and baryons (gas and stars) simultaneously, while SAMs evolve the baryons in a pre-computed (usually by an $N$-body simulation) dark matter background. These approaches are complementary: both approximate the key physical processes that are fundamental to galaxy formation, such as star formation and feedback, but hydrodynamical simulations directly solve the equations of gas dynamics and the gravitational interactions between the gas, stars and dark matter, whereas SAMs must approximate them. SAMs are computationally inexpensive, can model galaxy populations in cosmological volumes over cosmic time, and can be used to explore the parameter space of galaxy formation rapidly. In contrast, hydrodynamical simulations are computationally expensive but can capture the detailed spatial, kinematical and dynamical structure of gas, stars, and dark matter in galaxies within individual systems or in small volumes, and can be used to inform the choice of physically motivated recipes used in SAMs. Both approaches have shown substantial progress in the treatment of gas phases in and outside galaxies in the last $\sim 5$ years.

Hydrodynamical simulations have witnessed important advances in the treatment of the interstellar medium (ISM) in galaxies and in metal enrichment of the circumgalactic medium (CGM). Non-cosmological simulations of galaxy formation have been used to study in detail processes such as $\mathrm{H}_{2}$ formation and destruction, the $\mathrm{H} \mathrm{I}$ to $\mathrm{H}_{2}$ transition in non-equilibrium chemistry, nonequilibrium thermal state, and variations in the strength of the radiation field (e.g. Pelupessy et al. 2006, 2009; (Robertson \& Kravtsov (2008); Gnedin et al. (2009); Dobbs et al. (2011); Shetty \& Ostriker (2012); Glover \& Clark (2012); Christensen et al. (2012)). This work has allowed for physical interpretation of the observed relation between the SFR and the $\mathrm{H}_{2}$ mass surface densities observed in local galaxies. Another important area of progress has been in exploration of the mechanisms that drive turbulence in the ISM. (Dobbs et al. (2011) simulated a large spiral galaxy and showed that stellar feedback is important in setting the scale height of the galaxy disk through its effect on the velocity dispersion. Similarly, Shetty \& Ostriker (2012) find that supernovae are the primary source of turbulence in the dense environments of starburst galaxies. However, Bournaud et al. (2010) found in their simulation of a LMC-like dwarf galaxy that gravitational instabilities - which drive the velocity dispersion in their simulation - rather than stellar feedback set the scale height, while stellar feedback is necessary to maintain large-scale turbulence, which initiates cascades to small scales and suppresses the formation of very dense, small gas clumps (see also Hopkins et al. (2013) and Shetty \& Ostriker (2012)). In cosmological hydrodynamical simulations, the transition from $\mathrm{H}$ I to $\mathrm{H}_{2}$ has an important effect on the predicted number of large column density absorbers, $N_{\mathrm{H}}>7 \times 10^{21} \mathrm{~cm}^{-2}$ (Altay et al. (2011)). Extensive testing of the relation between galaxies and H I absorbers at high redshifts has been presented by Rahmati \& Schaye (2014), who reach con- 
clusions similar to SAMs (Lagos et al. (2011, 2014); Popping et al. (2014); Fu et al. (2012)), that a large fraction of the damped Ly- $\alpha$ absorbers are associated with galaxies with stellar masses $M_{\star}<10^{9} M_{\odot}$ (see also Davé et al. (2013); Bird et al. (2014); Rafieferantsoa et al. (2014)).

In semi-analytical models of galaxy formation, significant effort has focused on improving the modelling of the ISM in galaxies. Whereas previous generations of models followed the evolution of only a single cold gas phase (Obreschkow et al. (2009b); Power et al. (2010)), current models partition this cold gas phase into $\mathrm{H} \mathrm{I}$ and $\mathrm{H}_{2}$ phases and follow the evolution of these phases in a self-consistent manner (e.g. Cook et al. (2010); Fu et al. (2010); Lagos et al. (2011); Popping et al. (2014)). These models assume gas in hydrostatic and chemical equilibrium and use either the empirical relation of (Blitz \& Rosolowsky (2006)), which relates the $\mathrm{H}_{2} / \mathrm{H}$ I gas surface density ratio to the local hydrostatic pressure, or theoretical relations that calculate $\mathrm{H}_{2}$ abundance as a result of the equilibrium between rates of destruction due to UV radiation and formation on dust grains (e.g. (Krumholz et al. (2009); Gnedin \& Kravtsov (2011)). Models based on the empirical relation of Blitz \& Rosolowsky appear to provide a better fit to the $z=0 \mathrm{H} \mathrm{I}$ mass function (Zwaan et al. (2005); Martin et al. (2010)), with good agreement extending down to H I masses of $10^{6} M_{\odot} h^{-2}$ (Lagos et al. (2011), and also reproduce the observed clustering of $\mathrm{H}$ I selected galaxies (Kim et al. (2012)).

One of the key results of SAMs is shown in Fig. 1, which shows the very mild evolution of $\Omega_{H I}$ from $z=2$ to $z=0$. This prediction relates to a key question in extragalactic astrophysics: what accounts for the dramatically different evolution of the star formation rate (SFR) and $\mathrm{HI}$ densities of the Universe. Lagos et al. (2011) and Lagos et al. (2014) argue that the steep decline observed in the SFR density with decreasing redshift is closely connected to the steep decline of the molecular gas density - because star formation is linked explicitly to the $\mathrm{H}_{2}$ density in galaxies - whereas the H I density is predicted to evolve very weakly with redshift. Lagos et al. explain this trend as arising from a combination of decreasing gas fractions and increasing galaxy sizes with decreasing redshift, both of which act to reduce gas surface density and the hydrostatic pressure of the disk, which controls the rate at which stars form. Therefore, the SFR density evolution reflects the evolution of the surface density of gas in the galaxies that dominate the SFR in the universe as a function of cosmic time (see also Obreschkow \& Rawlings (2009)). This interpretation also implies an increase in the molecular to dynamical mass ratios and $\mathrm{H}_{2} / \mathrm{H}$ I mass ratio with increasing redshift, which is consistent with observations (Geach et al. (2011); Tacconi et al. (2013)).

Recent progress in the simulation and modelling of galaxy formation has been rapid, and has emphasized the critical role $\mathrm{H}$ I plays in driving galaxy formation and evolution. This work has also highlighted how pivotal $H$ I surveys on the SKA and its pathfinders will be in revealing the full extent of this role, how they will make a crucial contribution to multi-wavelength surveys campaigns, and how they will provide - arguably - the most powerful tests of the predictions of our theories of galaxy formation and evolution. For example, simulations make clear predictions for the expected relation between galaxy properties (such as stellar mass and SFRs), environment (measured by halo mass and also clustering) and H I content (Lagos et al. 2011, 2014, Davé et al. (2013); Kim et al. (2013); Rahmati \& Schaye (2014)), as well as the clustering of H I selected galaxies at different $\mathrm{H}$ I masses and cosmic times. These measurements will be partially accessible with the ASKAP and MeerKAT pathfinders, but it will be with SKA1 that the most complete and powerful tests will be made. 


\subsection{Upcoming SKA pathfinder H I surveys}

Various H I surveys are planned for the SKA pathfinder instruments MeerKAT, ASKAP and APERTIF over the next 5-7 years, ranging from deep observations of particular galaxies or clusters to very wide-area, relatively shallow surveys to narrow, deep surveys. A summary of the science aims and specifications for those surveys focusing on galaxy evolution is presented in Table 1.

\begin{tabular}{|c|c|}
\hline \multicolumn{2}{|c|}{$\begin{array}{l}\text { BLIND H I SURVEYS: WIDE-AREA vs. DEEP } \\
\text { Main aims: To investigate: } \\
\text { - Evolution of H I content (H I mass function) of galaxies vs. } z \text { and environment } \\
\text { - Galaxy formation \& missing satellite problem in Local Group } \\
\text { - Correlation of H I properties of galaxies and with stellar/halo masses, star-formation rates, etc. } \\
\text { - Physics governing cool gas distribution \& evolution at low } z \\
\text { - Baryonic Tully-Fisher relation vs. } z \\
\text { - Cosmological parameters relating to gas-rich galaxies } \\
\text { - Cosmic web }\end{array}$} \\
\hline CHILES (JVLA) [ongoing $]$ & Specifications: Single pointing in COSMOS field, for $z<0.45$ \\
\hline $\begin{array}{lc}\text { Medium-deep } & \text { blind imaging } \\
\text { (APERTIF) } & \text { [proposed }]\end{array}$ & Specifications: $z<0.25$ over $500 \mathrm{deg}^{2}$ \\
\hline $\begin{array}{l}\text { Northern sky H I shallow sur- } \\
\text { vey (APERTIF) [proposed] }\end{array}$ & Specifications: $\delta>+27^{\circ}, 0<z<0.26$ \\
\hline $\begin{array}{l}\text { WALLABY } \\
\text { [approved }]\end{array}$ & Specifications: $-90^{\circ}<\delta<+30^{\circ}, z<0.26$ \\
\hline DINGO (ASKAP) [approved] & $\begin{array}{l}\text { Specifications: } 2 \text { Phases: } \\
\text { DEEP: } z<0.26 \text { over } 150 \mathrm{deg}^{2} \\
\text { UDEEP: } 0.1<z<0.43 \text { over } 60 \mathrm{deg}^{2}\end{array}$ \\
\hline $\begin{array}{l}\text { LADUMA } \\
\text { [approved] }\end{array}$ & $\begin{array}{l}\text { Specifications: Single pointing encompassing ECDF-S, } z<1.4 \\
\text { over } \sim 4 \mathrm{deg}^{2},\end{array}$ \\
\hline
\end{tabular}

\section{H I SURVEYS OF INDIVIDUAL GALAXIES / CLUSTERS}

Main aims: To investigate:

- The range of conditions in ISM in local galaxies

- The gas-cycle in nearby galaxies by probing high vs. low column density regions

- The connection to local cosmic web

- Gas-stripping processes in clusters

- H I content vs. galaxy morphological type

\begin{tabular}{|l|l|}
\hline \hline MHONGOOSE (MeerKAT) & Specifications: 30 nearby galaxies with $10^{5}<M_{H I}<10^{10} M_{\odot}$, \\
approved $]$ & $N_{H I}(5 \sigma) \sim 10^{19} \mathrm{~cm}^{-2}$ \\
\hline $\begin{array}{l}\text { Fornax H I survey (MeerKAT) } \\
\text { [approved }]\end{array}$ & Specifications: Fornax cluster galaxies over $11 \mathrm{deg}^{2}$ down to \\
\hline
\end{tabular}

Table 1: Overview of upcoming H I emission line surveys on SKA pathfinder facilities, APERTIF [Medium-deep blind imaging survey, Northern sky H I shallow survey (Verheijen et al. (2009)) ], ASKAP [WALLABY and DINGO] (Duffy et al. (2012)) and MeerKAT [LADUMA (Holwerda et al. (2012)), MHONGOOSE (mhongoose.astron.nl) and the Fornax H I survey (Serra (2011))]. 
This table is meant to be a summary of H I line work to be carried out in the next 5-10 years which will form a solid basis for further work (more detailed and sensitive studies of selected objects and areas and pushing knowledge of $\mathrm{H} \mathrm{I}$ in and around galaxies to higher redshifts with the increased collecting area of SKA1 and eventually the SKA2). The SKA2 will not only be capable of providing optical quality ( $\sim 1$ " resolution) $\mathrm{H}$ I images of Milky Way size galaxies at $z=1$, but will also have the survey speed to carry out the billion galaxy survey that will chart out the large scale structure of the Universe from $z=0$ to $z=3$ and allow precision cosmology studies (Myers et al. (2009)).

\section{Probing galaxy evolution with the SKA}

Once SKA1 comes online, the various surveys mentioned above will have pushed our knowledge of $\mathrm{H} \mathrm{I}$ in galaxies and its role in galaxy evolution to a situation where we have a good inventory of detailed knowledge of the nearby universe. Shallow surveys will have covered most of the sky out to $z \sim 0.25$ (Duffy et al. (2012)) and provided H I masses of half a million galaxies, of which several tens of thousands of galaxies will be reasonably well resolved. Deeper surveys will have pushed to $z \sim 0.6$, but lack the detailed information needed to characterize the physical processes that govern galaxy evolution from the H I point of view: gas accretion, gas removal by winds, ram pressure or tidal stripping. Such details are also an important ingredient for comparisons with and input to the advanced modelling described in section 2.2. The obvious next steps are to obtain more sensitive and more detailed observations in the nearby universe $(0<z<0.25)$ for characterizing these processes and to push single-galaxy $\mathrm{HI}$ mass detections to larger distances and lower $\mathrm{HI}$ masses to explore the dependence on cosmic time and environment of the $\mathrm{H}$ I mass function and other scaling relations. In the following subsections, we briefly describe these science goals in more detail.

\subsection{The $\mathrm{H}$ I mass function}

The H I mass function (Rao \& Briggs (1993)) is one of the most important statistical measures for gas-rich galaxy populations. It describes the volume density of galaxies as a function of $\mathrm{HI}$ mass and is therefore the neutral hydrogen equivalent of the optical luminosity function. One important use of the H I mass function in the context of galaxy evolution is its second moment, which gives the cosmic $\mathrm{H}$ I mass density: the number of neutral hydrogen atoms per unit volume. This is an important input into the understanding of the evolution of the cosmic star formation rate density. When combined with damped Ly $\alpha$ measurements at higher redshifts (e.g., Noterdaeme et al. 2012, and references therein), the evolution of the H I mass density can be charted and compared to models of gas and galaxy evolution. The $\mathrm{H}$ I mass density can be compared with the predictions of semi-analytic or hydrodynamic models in order to study the processes governing the distribution and evolution of cool gas (e.g., Obreschkow \& Rawlings (2009), Lagos et al. 2011).

Of equal importance is the shape of the H I mass function. For example, Kim et al. (2012) demonstrate that the $\mathrm{HI}$ mass function is a more sensitive probe of cosmological reionization for galaxy formation than the optical galaxy luminosity function. For semi-analytic models, the H I mass function shape at $z=0$ is an indispensable constraint (Obreschkow \& Rawlings (2009), Lagos et al. 2010, Neistein et al. 2010, Kim et al. 2011). The H I mass function puts additional limits 
on the models compared to, e.g., the stellar mass function because the correlation between halo mass and cold gas mass is very different from that between halo mass and stellar mass (the most gas-rich galaxies often have low halo mass). Furthermore, Kim et al. (2012) show how different star formation laws affect the shape of the H I mass function.

The H I mass function has been well measured for the Local Universe (Zwaan et al. (2005); Martin et al. (2010); however, controversy still surrounds the magnitude and sign of the environmental density dependence of the $\mathrm{H}$ I mass function, possibly due to depth and cosmic variance issues with existing shallow surveys (Zwaan et al. (2005); Springob et al. (2005)). Upcoming precusor surveys such as WALLABY, will detect galaxies over a $\sim 100$ times larger volume and will therefore be much less sensitive to cosmic variance, enabling detailed study of the variation of the $\mathrm{HI}$ mass function with environmental density. The deeper HI pathfinder surveys such as DINGO and LADUMA will enable measurement of the $\mathrm{H}$ I mass function to intermediate redshifts, $z<0.6$.

With the SKA, it will be possible to push measurements of the $\mathrm{H}$ I mass function to even higher redshifts $(z \sim 1)$, which will in turn place even more stringent constraints on galaxy evolution models (see Fig. 2). It will also allow us to probe the evolution of the cosmic neutral gas density, $\Omega_{\text {gas }}$, from H I emission into the redshift range where it is currently estimated using damped Ly- $\alpha$ and magnesium absorber measurements (Prochaska et al. (2005); Rao et al. (2006); Noterdaeme et al. (2009, 2012); Zafar et al. (2013)).

Many questions demanding measurements of the H I masses of galaxies can also be investigated with these observations. As functions of both environment (i.e., field vs group or cluster)(e.g., Hess \& Wilcots (2013); Jaffé et al. (2013)) and cosmic time, we will be able to study, for lower H I masses than ever before, the relationships between H I mass and stellar mass, star formation rate, galaxy morphology, etc., as a means to uncover how galaxies evolve over time in different environments.

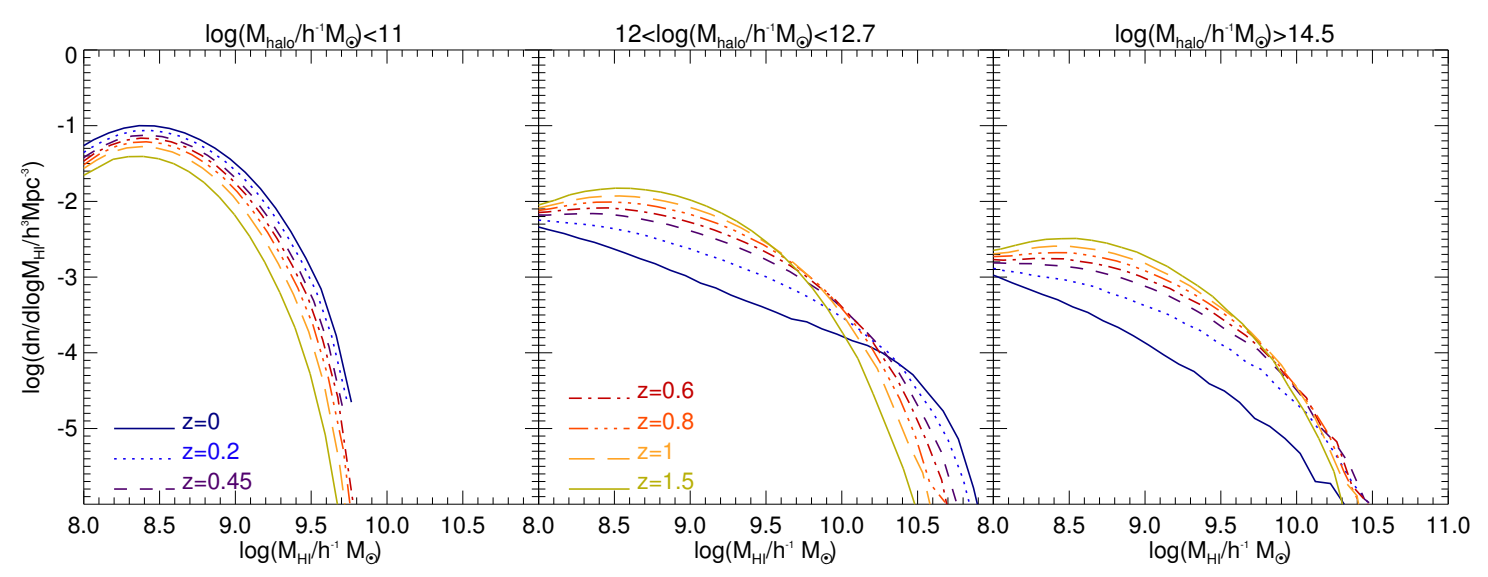

Figure 2: Models from Lagos et al. (2012) showing evolution of the H I mass function with redshift and halo mass. Halo mass bins correspond to field (left panel), group (middle panel), and cluster (right panel) environments. 


\subsection{Using $\mathrm{H}$ I to probe gas inflow and removal}

Galaxies form and evolve via the acquisition of gas from the intergalactic medium (IGM). In star-forming (blue cloud) galaxies, gas infall is expected to occur over a Hubble time to keep them forming stars at a relatively high rate (Fraternali \& Tomassetti (2012)). Indirect evidence for gas infall comes from estimates of gas depletion times (Bigiel et al. (2011)), the reconstruction of star formation histories (Panter et al. (2007)), and chemical evolution models of the Milky Way (Schönrich \& Binney (2009). However, at present the observational evidence for gas inflow into galaxies is rather scant, and it is not clear how and in what form it takes place (Sancisi et al. (2008)).

Inflow of gas into galaxies has a long history dating back to the discovery of the high-velocity clouds (HVCs) in the Milky Way (Wakker \& van Woerden (1997)). To date, the origin of these clouds remain a mystery, and their contribution to the feeding of the Galactic star formation is uncertain (Putman, et al. (2012)). Deep HI observations of external galaxies also show similar features in HI (Westmeier et al. (2005)). Fig. 3 shows examples of massive clouds/filaments observed in projection over the bright optical disk of three nearby galaxies. These filaments/complexes are well separated in velocity from the $\mathrm{H}$ I in their disks. These structures have $\mathrm{H}$ I masses similar to that of the Galactic HVC Complex C. It is unlikely that they result from a galactic fountain and they may be the signs of either minor mergers or the infall of cosmological filaments (Fernández, Joung, \& Putman (2012)).

The typical sensitivities achieved with the current interferometers for deep HI observations like those of NGC 2403 (Fraternali et al. (2002)) are of order $10^{19} \mathrm{~cm}^{-2}$. SKA will improve this sensitivity by 2 orders of magnitude, keeping the spatial resolution very high. This will have a tremendous impact on our understanding of gas inflow, as we will finally be able to trace the origin of these structures and detect gas falling into galaxies from the IGM. At present, the only information about low column density material around galaxies comes from absoption line studies towards AGNs. From the study of Ly $\alpha$ absorbers, it is clear that there are vast reservoirs of cold gas surrounding both star-forming and early type galaxies (Tumlinson et al. (2013)). However their origin, morphology, and fate are not clear; for instance whether they are gas clouds falling into the galaxies or simply floating in the galactic coronae remains ambiguous. This can be explored with the SKA.

Galaxies also eject gas from their disks as a consequence of stellar winds and supernova explosions. The ejected gas is likely multiphase but a large fraction of it is likely cold (Melioli et al. (2009)) or will cool rapidly (Houck \& Bregman (1990)). Observations show HI at large distance from the Milky Way's disk associated with superbubble expansion (Pidopryhora et al. (2007)) and gas at high velocities escaping from holes in the disks of external galaxies (Boomsma et al. (2008)).

Such HI outflows produce large layers of extra-planar gas that contain typically $10 \%$ of the HI mass of a galaxy, reaching up to 25\% in exceptional cases (Oosterloo et al. (2007); Sancisi et al. (2008)). The study of the kinematics of $\mathrm{H}$ I halos gives essential information about the exchange of material and angular momentum between galactic disks and the IGM (Marinacci et al. (2010)). However, at the moment, the overall picture is not understood, as these low column density features are not seen around all galaxies and in several cases are not very extended (Heald et al. (2011)). Going down to a sensitivity well below $\times 10^{18} \mathrm{~cm}^{-2}$ will provide unprecented information about gas escaping from galaxies and how this interacts with the surrounding medium. With SKA2 we 

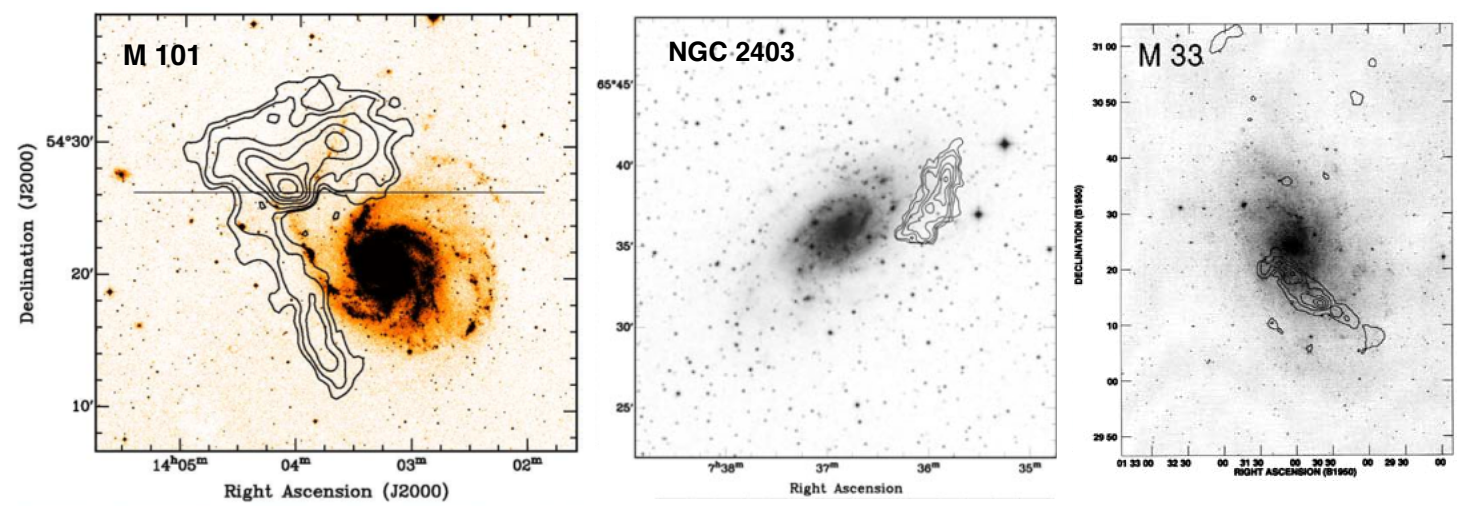

Figure 3: Three examples of possible accretion of $\mathrm{HI}$ in nearby spirals indicated by the presence of gas complexes at anomalous velocities: M 101 (van der Hulst \& Sancisi (1988)), NGC 2403 (Fraternali et al. (2002)), and M 33 (Sancisi et al. (2008)).

will eventually be able to fully trace the gas cycle from disks to the IGM and understand its crucial role for the evolution of star-forming galaxies.

Gas can also be removed from galaxies via tidal interactions (Sancisi et al. (2008)) and ram pressure stripping in galaxy clusters (Kenney et al. (2004), see also Fig. 4). At the moment, these observations are limited to very few cases of mostly very massive structures (Oosterloo \& van Gorkom (2005)) although H I deficiencies are also found in small but dense environments, such as Hickson compact groups (Verdes-Montenegro et al. (2001)). SKA will change this dramatically, giving the possibility to study tidal interactions and minor mergers in unprecentedly large galaxy samples. We will be able to investigate the prevalence of these phenomena, the accretion and removal rates from interaction and how all these properties change as a function of environment and time. The study of gas removed from galaxies in dense environments will also finally solve the problem of star formation quenching, something that is considered of fundamental importance for the migration of star-forming galaxies towards the red sequence (Blanton \& Moustakas (2009)).

\subsection{The TF relation}

The Tully-Fisher relation (TFr), originally describing a correlation between a galaxy's intrinsic luminosity and the distance-independent width of its global H I profile (Tully \& Fisher (1977)), is the most important observed scaling relation for gas-rich, late-type galaxies. Although the TFr is empirical in nature, it seems astrophysically plausible that a galaxy's luminosity, as a proxy for its stellar mass, should be related to its gravitational potential as traced by the rotation speed of its gas disk, observed through the Doppler-broadened width of its H I emission line. The presence of dark matter and the detailed distribution of the visible mass inside galaxies complicate this naive interpretation (Zwaan et al. (1995)). Nevertheless, the TFr serves as a valuable tool to obtain distances to galaxies in the local universe where peculiar velocities dominate over the Hubble flow (Tully \& Courtois (2012)), while its statistical properties constrain semi-analytical and hydrodynamical simulations of galaxy formation and evolution. Studies of the detailed H I kinematics of regularlyrotating galaxies for which the extended $\mathrm{H}$ I gas disk is a proper tracer of the gravitational potential, and for which good estimates of their relative stellar masses are available, indicate that the TFr is 
fundamentally a correlation between the maximum rotational velocity of the dark matter halo and the embedded total baryonic mass, regardless of how this baryonic mass is distributed (McGaugh et al. (2000); Verheijen (2001); McGaugh (2005)).

Major advances in photometric imaging technologies have significantly improved our measures and understanding of a galaxy's luminosity to an extent that, combined with spectroscopic information, it has become common practice to replace a galaxy's luminosity in the TFr by its derived stellar mass, or even its baryonic mass when taking the atomic and estimated molecular gas masses into account (McGaugh (2005); Zaritsky et al. (2014)). Improving our understanding of the width of the global $\mathrm{H}$ I line as a kinematic measure has received little attention so far, and even the most recent studies still use this quantity (Sorce et al. (2013); Zaritsky et al. (2014)). As galaxies are usually not spatially resolved by single-dish radio telescopes, the shape and width of the global H I profile is the result of a convolution of the detailed distribution of the H I gas in a galaxy with the detailed geometry and kinematics of the gas disk, including possible warps, streaming motions, asymmetries and the overall shapes of rotation curves, which may not always be monotonically rising to a constant circular velocity (Bosma (1981a,b); Begeman (1989); Casertano \& van Gorkom (1991); Verheijen \& Sancisi (2001); Noordermeer et al. (2007); Swaters et al. (2009)). Hence, the width of the global H I profile is only a proxy for the rotational speed of a galaxy, while the latter may not even be properly defined and identified for non-flat rotation curves, and trends in rotation curve shapes along the TFr may introduce significant biases in scatter and slope (Verheijen (2001); Noordermeer \& Verheijen (2007)). Imaging the geometry and kinematics of a galaxy's extended $\mathrm{H}$ I disk with interferometers is the only observational avenue to identify the relevant kinematic measure for the TFr and to significantly improve our understanding of the underlying astrophysics.

It is expected that upcoming $\mathrm{H}$ I imaging surveys with ASKAP, WSRT/APERTIF and MeerKAT will further reduce the observed scatter in the TFr by spatially resolving the H I kinematics thereby improving TF-based distances to galaxies in the local universe. These imaging surveys will also establish the statistical properties of the TFr at modest redshifts $(z<0.2)$ using spatially resolved H I kinematics, and up to $z<0.5$ using global H I line widths. It is unlikely that the SKA will significantly improve upon these studies. However, given the anticipated sensitivity and angular resolution of the SKA, it will be possible to exploit the TFr to study the evolution of galaxies over cosmic time by investigating the slope, scatter and zero-point as a function of redshift and cosmic environment, as well as global galaxy properties such as morphological type and (specific) star formation rate. This requires adequate angular resolution and column density sensitivity to image the H I kinematics in the outer gas disks of galaxies at higher redshifts, as well as survey volumes that encompass all cosmic environments with sufficient statistical significance to mitigate cosmic variance. In order to assess the kinematical state of the largest $\mathrm{H}$ I disks in $\mathrm{M}_{H I}^{*}$ galaxies at a redshift of $z=0.5$, a spatial resolution of $10 \mathrm{kpc}$ would be sufficient, corresponding to an angular resolution of 1.6 arcseconds at a frequency of $950 \mathrm{MHz}$. Based on studies of nearby galaxies, the required column density sensitivity at this angular resolution would need to be $\sim 5 \times 10^{20}\left[\mathrm{~cm}^{-2}\right]$. This could be achievable with an ultra-deep survey using SKA1-MID but seems out of reach for SKA-SUR. The volume surveyed by a single SKA1-MID pointing at $z=0.5$ at $950 \mathrm{MHz}$ is large enough to sample all cosmic environments with a sufficient number of detections to provide adequate statistics on the TFr. Pushing TFr studies based on resolved H I kinematics to even higher redshifts would require the power of SKA2, in particular given the required column density sensitivities. 


\section{SKA HI Strawman Surveys}

\subsection{Survey scenarios and requirements}

The science goals outlined in section 3 should lead us to define the most efficient observing strategies for obtaining the data, given the the technical capabilities of the SKA, in the first phase and eventual final form. A review of the science addressed in section 3 makes clear immediately that it is of the greatest importance not only to have a sufficiently large number of objects for statistical studies probing different regions of the universe, both spatially and in cosmic time, but also to have the appropriate linear resolution and column density sensitivity to probe the phenomena of interest. For studies involving the $\mathrm{H}$ I mass function, it is important to push the detection limit to objects with small $\mathrm{H}$ I masses $\left(<10^{7} \mathrm{M}_{\odot}\right)$ without having to resolve the $\mathrm{H}$ I distributions. For all other science goals it is extremely important to resolve the galaxies and be able to detect $\mathrm{H} \mathrm{I}$ column densities to levels well below $N_{\mathrm{HI}} \sim 10^{21} \mathrm{~cm}^{-2}$. This is beautifully illustrated in Fig. 4 .
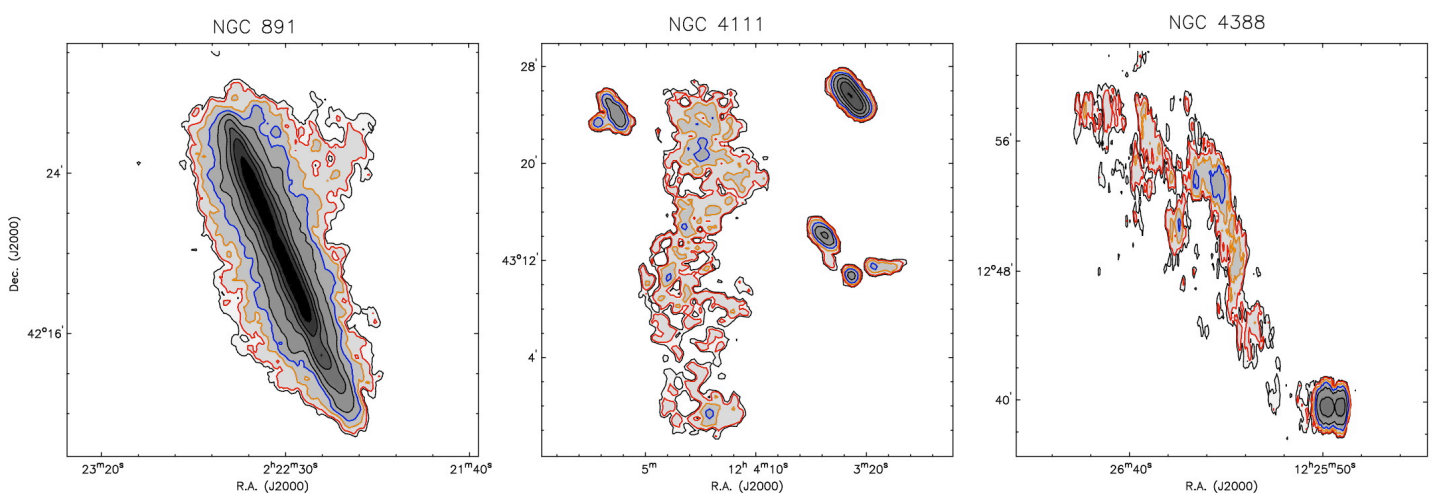

Figure 4: Three examples of galaxies undergoing gas accretion and gas removal events. From left to right: NGC891 (Oosterloo et al. (2007)), NGC4111 (Verheijen (2004)), and NGC4388 (Oosterloo \& van Gorkom (2005)). The lowest contours levels are $2 \times 10^{20} \mathrm{~cm}^{-2}$ (red), $5 \times 10^{20} \mathrm{~cm}^{-2}$ (orange) and $10 \times 10^{20} \mathrm{~cm}^{-2}$ (blue). It is clear that the signs of gas accretion (NGC891, NGC4111) and of gas stripping (NGC4388) require column density sensitivities of well below $10^{21} \mathrm{~cm}^{-2}$.

Using the specifications of the SKA baseline design (Dewdney (2013)), one can derive the observing parameters required to reach a depth of $N_{\mathrm{HI}} \sim 10^{20} \mathrm{~cm}^{-2}$ at reasonable resolution. Table 2 provides basic input to these considerations. The numbers are based on the latest sensitivity estimates for SKA1-MID, SKA1-SUR and SKA2. Table 2 provides information for four different resolutions because $N_{\mathrm{HI}}$ sensitivity scales with resolution. The corresponding linear resolution is given for two nominal distances. In addition, Table 2 gives the $\mathrm{H}$ m mass limits for a 10 hour integration assuming a profile width of $50 \mathrm{~km} \mathrm{~s}^{-1}$ for three nominal distances. For reference, the canonical value for $M_{\mathrm{HI}}^{*}$ is $\sim 8 \times 10^{9} \mathrm{M}_{\odot}$ (Zwaan et al. (2003, 2005); Haynes et al. (2011)).

It is clear that in order to design the right surveys for the science described in this chapter, trade-offs need to be made between resolution, integration time, and area covered. Table 3 provides an attempt to do this for SKA1. Survey areas are chosen such that the total integration time remains realistic, while the appropriate resolution and column density are chosen to match the science goals outlined in the previous sections. For example, a survey of the entire southern sky to a depth of 
Table 2: SKA sensitivity limits (5 sigma) for HI emission

SKA1-MID $t=10 \mathrm{~h}$

\begin{tabular}{|c|c|c|c|c|c|c|c|c|}
\hline $\begin{array}{l}\text { Resolution } \\
\text { ( " ) }\end{array}$ & $\begin{array}{c}\mathrm{d}(\mathrm{z}=0.2) \\
(\mathrm{kpc})\end{array}$ & $\begin{array}{c}\mathrm{d}(\mathrm{z}=0.5) \\
(\mathrm{kpc})\end{array}$ & $\begin{array}{c}\text { rms noise } \\
m J y \\
\mathrm{dV}=5 \mathrm{~km} / \mathrm{s}\end{array}$ & $\begin{array}{c}5 \text { sigma } N(H I) \\
d V=5 \mathrm{~km} / \mathrm{s}\end{array}$ & $\begin{array}{r}5 \text { sigma } N(H I) \\
d V=25 \mathrm{~km} / \mathrm{s}\end{array}$ & $\begin{array}{c}5 \text { sigma } M(\mathrm{HI}) \\
\mathrm{dV}=50 \mathrm{~km} / \mathrm{s} \\
\mathrm{z}=0.02\end{array}$ & $\begin{array}{c}5 \text { sigma } M(\mathrm{HI}) \\
\mathrm{dV}=50 \mathrm{~km} / \mathrm{s} \\
\mathrm{z}=0.2\end{array}$ & $\begin{array}{c}5 \text { sigma } M(\mathrm{HI}) \\
\mathrm{dV}=50 \mathrm{~km} / \mathrm{s} \\
\mathrm{z}=0.5\end{array}$ \\
\hline 1 & 3.3 & 6.1 & 0.141 & $3.92 \mathrm{E}+21$ & $1.96 \mathrm{E}+22$ & $1.20 \mathrm{E}+07$ & $1.56 \mathrm{E}+09$ & $1.32 \mathrm{E}+10$ \\
\hline 3 & 9.8 & 18.4 & 0.118 & $3.65 \mathrm{E}+20$ & $1.83 \mathrm{E}+21$ & $1.00 \mathrm{E}+07$ & $1.31 \mathrm{E}+09$ & $1.10 \mathrm{E}+10$ \\
\hline 10 & 32.8 & 61.2 & 0.091 & $2.53 E+19$ & $1.26 \mathrm{E}+20$ & $7.72 \mathrm{E}+06$ & $1.01 \mathrm{E}+09$ & $8.50 E+09$ \\
\hline 30 & 98.3 & 183.7 & 0.095 & $2.95 \mathrm{E}+18$ & $1.47 \mathrm{E}+19$ & $8.11 \mathrm{E}+06$ & $1.06 \mathrm{E}+09$ & $8.92 \mathrm{E}+09$ \\
\hline
\end{tabular}

SKA1-SUR $\quad t=10 h$

\begin{tabular}{|c|c|c|c|c|c|c|c|c|}
\hline $\begin{array}{c}\text { Resolution } \\
(\mathrm{l})\end{array}$ & $\begin{array}{c}\mathrm{d}(\mathrm{z}=0.2) \\
(\mathrm{kpc})\end{array}$ & $\begin{array}{c}\mathrm{d}(\mathrm{z}=0.8) \\
(\mathrm{kpc})\end{array}$ & $\begin{array}{c}\text { rms noise } \\
\text { mJy } \\
\mathrm{dV}=5 \mathrm{~km} / \mathrm{s}\end{array}$ & $\begin{array}{c}5 \text { sigma N(HI) } \\
d V=5 \mathrm{~km} / \mathrm{s}\end{array}$ & $\begin{array}{r}5 \text { sigma N(HI) } \\
d V=25 \mathrm{~km} / \mathrm{s}\end{array}$ & $\begin{array}{c}5 \text { sigma } M(\mathrm{HI}) \\
\mathrm{dV}=50 \mathrm{~km} / \mathrm{s} \\
\mathrm{z}=0.02\end{array}$ & $\begin{array}{c}5 \text { sigma } M(\mathrm{HI}) \\
\mathrm{dV}=50 \mathrm{~km} / \mathrm{s} \\
\mathrm{z}=0.2\end{array}$ & $\begin{array}{c}5 \text { sigma } M(\mathrm{HI}) \\
\mathrm{dV}=50 \mathrm{~km} / \mathrm{s} \\
\mathrm{z}=0.5\end{array}$ \\
\hline 1 & 3.3 & 6.1 & 0.551 & $1.06 \mathrm{E}+22$ & $5.28 \mathrm{E}+22$ & $4.67 \mathrm{E}+07$ & $6.11 \mathrm{E}+09$ & $5.14 \mathrm{E}+10$ \\
\hline 3 & 9.8 & 18.4 & 0.361 & $9.96 \mathrm{E}+20$ & $4.98 \mathrm{E}+21$ & $3.06 \mathrm{E}+07$ & $4.00 E+09$ & $3.37 E+10$ \\
\hline 10 & 32.8 & 61.2 & 0.323 & $1.00 \mathrm{E}+20$ & $5.01 E+20$ & $2.74 \mathrm{E}+07$ & $3.58 \mathrm{E}+09$ & $3.02 E+10$ \\
\hline 30 & 98.3 & 183.7 & 0.588 & $1.88 \mathrm{E}+19$ & $9.38 \mathrm{E}+19$ & $5.00 \mathrm{E}+07$ & $6.53 E+09$ & $5.50 E+10$ \\
\hline
\end{tabular}

SKA2 $t=10 h$

\begin{tabular}{|c|c|c|c|c|c|c|c|c|}
\hline $\begin{array}{l}\text { Resolution } \\
\text { ( " ) }\end{array}$ & $\begin{array}{c}\mathrm{d}(\mathrm{z}=0.2) \\
(\mathrm{kpc})\end{array}$ & $\begin{array}{c}\mathrm{d}(\mathrm{z}=0.8) \\
(\mathrm{kpc})\end{array}$ & $\begin{array}{c}\text { rms noise } \\
m J y \\
d V=5 \mathrm{~km} / \mathrm{s}\end{array}$ & $\begin{array}{c}5 \text { sigma } \mathrm{N}(\mathrm{HI}) \\
\mathrm{dV}=5 \mathrm{~km} / \mathrm{s}\end{array}$ & $\begin{array}{c}5 \text { sigma N(HI) } \\
d V=25 \mathrm{~km} / \mathrm{s}\end{array}$ & $\begin{array}{c}5 \text { sigma } M(H I) \\
d V=50 \mathrm{~km} / \mathrm{s} \\
z=0.02\end{array}$ & $\begin{array}{c}5 \text { sigma } \mathrm{M}(\mathrm{HI}) \\
\mathrm{dV}=50 \mathrm{~km} / \mathrm{s} \\
\mathrm{z}=0.2\end{array}$ & $\begin{array}{c}5 \text { sigma } M(\mathrm{HI}) \\
\mathrm{dV}=50 \mathrm{~km} / \mathrm{s} \\
\mathrm{z}=0.5\end{array}$ \\
\hline 1 & 3.3 & 6.1 & 0.009 & $2.53 \mathrm{E}+20$ & $1.26 \mathrm{E}+21$ & $7.64 \mathrm{E}+05$ & $9.99 \mathrm{E}+07$ & $8.41 \mathrm{E}+08$ \\
\hline 3 & 9.8 & 18.4 & 0.009 & $2.81 \mathrm{E}+19$ & $1.40 \mathrm{E}+20$ & $7.64 \mathrm{E}+05$ & $9.99 \mathrm{E}+07$ & $8.41 E+08$ \\
\hline 10 & 32.8 & 61.2 & 0.009 & $2.53 E+18$ & $1.26 \mathrm{E}+19$ & $7.64 \mathrm{E}+05$ & $9.99 \mathrm{E}+07$ & $8.41 E+08$ \\
\hline 30 & 98.3 & 183.7 & 0.009 & $2.81 E+17$ & $1.40 \mathrm{E}+18$ & $7.64 \mathrm{E}+05$ & $9.99 E+07$ & $8.41 \mathrm{E}+08$ \\
\hline
\end{tabular}

Table 3: Strawman SKA1 surveys

\begin{tabular}{|c|c|c|c|c|}
\hline & Shallow survey & Medium deep survey & Deep survey & Ultra Deep Survey \\
\hline Area & $10000 \mathrm{deq}^{2}$ & $2000 \mathrm{deq}^{2}$ & $150 \mathrm{deq}^{2}$ & $2 \mathrm{deq}^{2}$ \\
\hline Integration time & 1 yr (Sur) & 1 yr (Sur) & 1 yr (Sur) & 1 yr (Mid) \\
\hline Resolution & $10 "$ & $5 "$ & 3" & $2 "$ \\
\hline$z\left(M_{\mathrm{HI}} *\right)$ & 0.27 & 0.37 & 0.69 & 1.7 \\
\hline mean $z$ & 0.08 & 0.11 & 0.19 & 0.37 \\
\hline nr. of detections & $2.02 E+06$ & $1.20 \mathrm{E}+06$ & $4.60 \mathrm{E}+05$ & $4.20 E+04$ \\
\hline
\end{tabular}

$N_{\mathrm{HI}} \sim 10^{20}$ at a resolution of $2 "$ would take half a century with SKA1, so a better approach is to cover a smaller area at lower but still useful resolution, leaving substantial improvement on a large H I survey such as WALLABY to SKA2. Which surveys cover substantial gound in redshift space is very well illustrated in Fig. 5, showing the distribution over redshift of the four surveys from Table 3. The distributions represent five statistically independent realisations of a $100 \mathrm{deg}^{2}$ sky model, truncated to $z=1.2$, based on the S3-SAX simulation (Obreschkow et al. (2009a,b)) using the telescope simulations of Popping et al. (2014) which are consistent with the sensitivity calculations used here. In other words SKA1 is the appropriate instrument for addressing followup science from the SKA pathfinders; SKA2 provides a major step towards pushing our observing capabilities to higher redshifts, i.e., look back times. In order to obtain a good census of galaxy evolution and the influence of the environment over cosmic time, one has to design surveys in such a way that SKA1 probes the epochs $0.1<z<0.5$ with similar linear resolution and $N_{\mathrm{HI}}$ sensitivity as APERTIF, ASKAP and MeerKAT will probe at $0.02<z<0.2$, while SKA2 will eventually provide the data required to get the full picture of $\mathrm{HI}$ evolution from the current epoch to $z \sim 2$ when the star formation rate density in the evolving universe is at its peak.

\section{Summary and conclusions}

Our current H I view of the universe is restricted to the Local Volume and hence only informs 


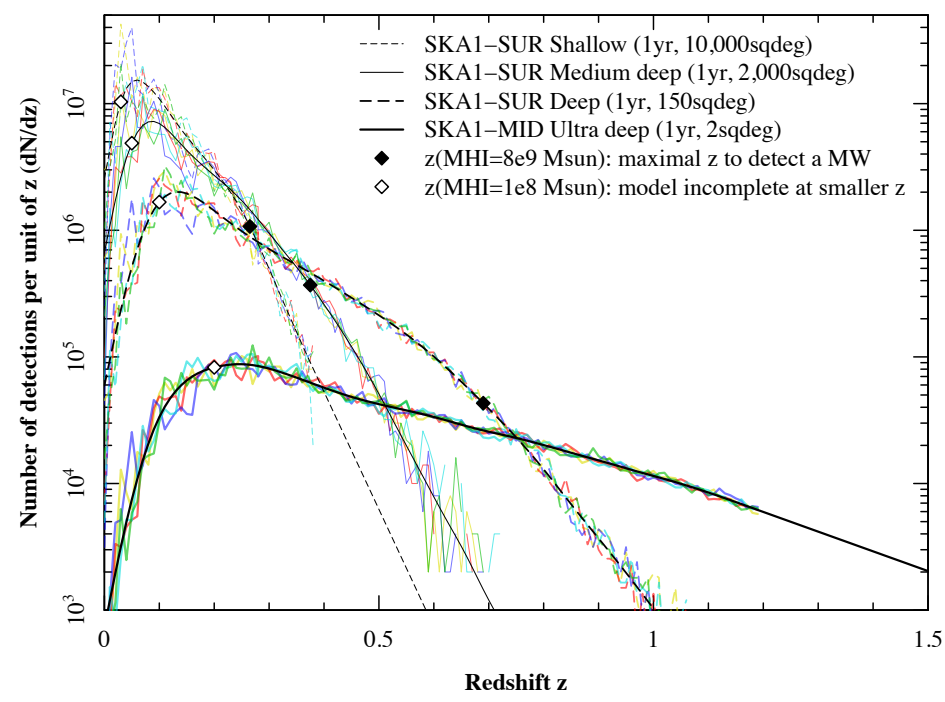

Figure 5: Distribution over redshift of the four surveys given in Table 3 based on the S3-SAX simulation (Obreschkow et al. (2009a,b))

us about gas-related processes in galaxies in a late state of their cosmic evolution. Semi-analytical and hydrodynamical models, however, suggest significant changes in the H I properties of galaxies beyond the Local Universe. We are poised to start testing these models with upcoming surveys on the SKA pathfinder instruments over the next few years. These facilities will provide much improved statistics on the $\mathrm{HI}$ properties of galaxies and will begin to open a window on the $\mathrm{HI}$ universe beyond the Local Volume. The SKA1 (and eventually SKA2) will truly revolutionise studies of $\mathrm{HI}$ in galaxies by enabling observations at higher angular resolutions and with sensitivities that are an order of magnitude greater than currently possible. Most importantly, the SKA1 will allow us to reach out to distances and lookback times spanning more than half the age of the universe. Combined with data at other wavelengths and detailed theoretical models, H I observations with the SKA1 will enable us to trace the baryon cycle in galaxies from the IGM and back out again in different environments. The SKA2 offers the exciting prospect of directly measuring the HI masses of the nearest Lyman limit systems and performing precision cosmology with the billion galaxy survey. Ultimately, we will reveal the evolution of the distribution and kinematics of gas in galaxies back to $z \sim 2$ when the cosmic star formation rate density was at its peak and an order of magnitude higher than the present, thereby building a comprehensive picture of galaxy evolution over cosmic time.

\section{Acknowledgments}

We thank various colleagues for valuable input to this chapter. JMvdH acknowledges support from the European Research Council under the European Union's Seventh Framework Programme (FP/2007-2013) / ERC Grant Agreement nr. 291531. BC is the recipient of an Australian Research Council Future Fellowship (FT120100660). MPH is supported by US-NSF/AST-1107390 and the Brinson Foundation. LVM has been supported by grants AYA2011-30491-C02-0S1 and TIC-114. 


\section{References}

Acreman, D. M., Dobbs, C. L., Brunt, C. M., \& Douglas, K. A. 2012, MNRAS, 422, 241

Altay, G., Theuns, T., Schaye, J., Crighton, N. H. M., \& Dalla Vecchia, C. 2011, ApJ, 737, L37

Baldry, I. K., Glazebrook, K., Brinkmann, J., et al., 2004, ApJ, 600, 681

Barnes, D. G., Staveley-Smith, L., de Blok, W. J. G., et al., 2001, MNRAS, 322, 486

Begeman, K. G. 1989, A\&A, 223, 47

Begum, A., Chengalur, J. N., Karachentsev, I. D., Sharina, M. E., \& Kaisin, S. S., MNRAS, 386, 1667

Beygu, B., Kreckel, K., van de Weygaert, R., van der Hulst, J. M., \& van Gorkom, J. H., AJ, 145, 120

Bigiel, F., Leroy, A., Walter, F., et al. 2008, AJ, 136, 2846

Bigiel, F., Leroy, A. K., Walter, F., et al., ApJ, 730, 2011, 13

Bird, S., Vogelsberger, M., Haehnelt, M., et al. 2014, MNRAS, 44, 2313

Blanton, M. R. and Moustakas, J., 2009, ARA\&A, 47, 159

Blitz, L., \& Rosolowsky, E. 2006, ApJ, 650, 933

Borthakur, S., Yun, M. S., \& Verdes-Montenegro, L., 2011, ApJ, 729, 149

Bosma, A., 1981, AJ, 86, 1791

Bosma, A., 1981, AJ, 86, 1825

Bournaud, F., Elmegreen, B. G., Teyssier, R., Block, D. L., \& Puerari, I. 2010, MNRAS, 409, 1088

Boomsma, R., Oosterloo, T. A., Fraternali, F., van der Hulst, J. M., and Sancisi, R., 2008, A\&A, 490, 555

Calzetti, D., Liu, G., \& Koda, J. 2012, ApJ, 752, 98

Cannon, J. M., Giovanelli, R., Haynes, M. P., et al., 2011, ApJ, 739, L22

Casertano, S., \& van Gorkom, J. H., 1991,AJ, 101, 1231

Catinella, B., Haynes, M. P., Giovanelli, R., Gardner, J. P., \& Connolly, A. J. 2008, ApJ, 685, L13

Catinella, B., Schiminovich, D., Kauffmann, G., et al., 2010, MNRAS, 403, 683

Chengalur, J. N., Braun, R., \& Wieringa, M. 2001, A\&A, 372, 768

Christensen, C., Quinn, T., Governato, F., et al. 2012, MNRAS, 425, 3058

Chung, A., van Gorkom, J. H., Kenney, J. D. P., Crowl, H., \& Vollmer, B., 2009, AJ, 138, 1741

Cook, M., Evoli, C., Barausse, E., Granato, G. L., \& Lapi, A. 2010, MNRAS, 402, 941

Cowie, L. L., Songaila, A., Hu, E. M., \& Cohen, J. G. 1996, AJ, 112, 839

Davé, R., Katz, N., Oppenheimer, B. D., Kollmeier, J. A., \& Weinberg, D. H. 2013, MNRAS, 434, 2645

de Blok, W.J.G., Fraternali, F., Heald, G.H., Adams, E.A.K., Bosma, A., Koribalski, B.S. \& the H I Science Working Group, "The SKA view of the Neutral Interstellar Medium in Galaxies", in proc. Advancing Astrophysics with the Square Kilometre Array, PoS(AASKA14)129

Delhaize, J., Meyer, M. J., Staveley-Smith, L., \& Boyle, B. J. 2013, MNRAS, 433, 1398

Dewdney, P., Turner, W., Millenaar, R., et al., 2013, SKA1 System Baseline Design, Document number SKA-TEL-SKO-DD-001 Revision 1

Dobbs, C. L., Burkert, A., \& Pringle, J. E. 2011, MNRAS, 417, 1318

Duffy, A. R., Meyer, M. J., Staveley-Smith, L., et al., 2012, MNRAS, 426, 3385

Duffy, A. R., Kay, S. T., Battye, R. A., et al., 2012, MNRAS, 420, 2799 
Espada, D., Verdes-Montenegro, L., Huchtmeier, W. K., et al., 2011, A\&A, 532, A117

Fabello, S., Catinella, B., Giovanelli, R., et al. 2011, MNRAS, 411, 993

Fabello, S., Kauffmann, G., Catinella, B., et al. 2012, MNRAS, 427, 2841

Feldmann, R., Gnedin, N. Y., \& Kravtsov, A. V. 2011, ApJ, 732, 115

Fernandez, X., Joung, M. R., and Putman, M. E., 2012, ApJ, 749, 181

Fernández, X., van Gorkom, J. H., Hess, K. M., et al., 2013, ApJ, 770, L29

Fu, J., Guo, Q., Kauffmann, G., \& Krumholz, M. R. 2010, MNRAS, 409, 515

Fraternali, F., van Moorsel, G., Sancisi, R., \& Oosterloo, T., 2002, AJ, 123, 3124

Fraternali, F. and Binney, J. J., 2008, MNRAS, 386, 935

Fraternali, F. and Tomassetti, M., 2012, MNRAS, 426, 2166

Freudling, W., Staveley-Smith, L., Catinella, et al., 2011, ApJ, 727, 40

Fu, J., Kauffmann, G., Li, C., \& Guo, Q. 2012, MNRAS, 424, 2701

Geach, J. E., Smail, I., Moran, S. M., et al. 2011, ApJ, 730, L19

Geréb, K., Morganti, R., Oosterloo, T. A., Guglielmino, G., \& Prandoni, I., 2013, A\&A, 558, A54

Giovanelli, R., Haynes, M. P., Adams, E. A. K., et al., 2013, AJ, 146, 15

Giovanelli, R., Haynes, M. P., Kent, B. R., et al., 2005, AJ, 130, 2598

Glover, S. C. O., \& Clark, P. C. 2012, MNRAS, 421, 9

Gnedin, N. Y., Tassis, K., \& Kravtsov, A. V. 2009, ApJ, 697, 55

Gnedin, N. Y., \& Kravtsov, A. V. 2011, ApJ, 728, 88

Haynes, M. P., Giovanelli, R., Martin, A. M., et al., 2011, AJ, 142, 170

Heald, G., Józsa, G., Serra, P., et al., 2011, A\&A, 526, A118

Hess, K. M., \& Wilcots, E. M. 2013, AJ, 146, 124

Holwerda, B. W., Blyth, S.-L., \& Baker, A. J., 2012, IAU Symposium, 284, 496

Hopkins, A. M., \& Beacom, J. F. 2006, ApJ, 651, 142

Hopkins, P. F., Hernquist, L., Cox, T. J., Dutta, S. N., \& Rothberg, B. 2008, ApJ, 679, 156

Hopkins, P. F., Narayanan, D., Murray, N., \& Quataert, E. 2013, MNRAS, 433, 69

Houck, J. C. and Bregman, J. N., 1990, ApJ, 352, 506

Hunter, D. A., Ficut-Vicas, D., Ashley, T., et al., 2012, AJ, 144, 134

Jaffé, Y. L., Poggianti, B. M., Verheijen, M. A. W., Deshev, B. Z., \& van Gorkom, J. H., 2013, MNRAS, 431, 2111

Jaffé, Y. L., Poggianti, B. M., Verheijen, M. A. W., Deshev, B. Z., \& van Gorkom, J. H., 2012, ApJ, 756, L28

Kenney, J. D. P., van Gorkom, J. H., and Vollmer, B., 2004, AJ, 127, 3361

Kim, H.-S., Lacey, C. G., Cole, S., et al. 2012, MNRAS, 425, 2674

Kim, H.-S., Power, C., Baugh, C. M., et al. 2013, MNRAS, 428, 3366

Koribalski, B. S., 2008, Galaxies in the Local Volume, 41

Kreckel, K., Platen, E., Aragón-Calvo, M. A., et al., 2012, AJ, 144, 16

Krumholz, M. R., McKee, C. F., \& Tumlinson, J. 2009, ApJ, 699, 850

Lagos, C. D. P., Baugh, C. M., Lacey, C. G., et al. 2011, MNRAS, 418, 1649

Lagos, C. D. P., Lacey, C. G., Baugh, C. M., Bower, R. G., \& Benson, A. J. 2011, MNRAS, 416, 1566

Lagos, C. d. P., Bayet, E., Baugh, C. M., et al. 2012, MNRAS, 426, 2142

Lagos, C. D. P., Baugh, C. M., Zwaan, M. A., et al. 2014, MNRAS, 440, 920 
Lah, P., Chengalur, J. N., Briggs, F. H., et al. 2007, MNRAS, 376, 1357

Lah, P., Pracy, M. B., Chengalur, J. N., et al. 2009, MNRAS, 399, 1447

Leroy, A. K., Bigiel, F., de Blok, W. J. G., et al. 2012, AJ, 144, 3

Madau, P., Pozzetti, L., \& Dickinson, M. 1998, ApJ, 498, 106

Marinacci, F., Binney, J., Fraternali, F., Nipoti, C., Ciotti, L., and Londrillo, P., 2010, MNRAS, 404, 1464

Martin, A. M., Papastergis, E., Giovanelli, R., et al. 2010, ApJ, 723, 1359

McGaugh, S. S., Schombert, J. M., Bothun, G. D., \& de Blok, W. J. G., 2000, ApJ, 533, L99

McGaugh, S. S., 2005, ApJ, 632, 859

Melioli, C., Brighenti, F., D'Ercole, A., and de Gouveia Dal Pino, E. M., 2009, MNRAS, 399, 1089

Meyer, M. J., Zwaan, M. A., Webster, R. L., et al., 2004, MNRAS, 350, 1195

Morganti, R., Sadler, E., Curran, S.J. \& the HI Science Working Group, "Cool Outflows and HI absorbers with SKA", in proc. Advancing Astrophysics with the Square Kilometre Array, PoS(AASKA14)134

Myers, S. T., Abdalla, F. B., Blake, C., et al., 2009, Astro2010: The Astronomy and Astrophysics Decadal Survey, Science White Papers, no. 219

Noordermeer, E., van der Hulst, J. M., Sancisi, R., Swaters, R. A., \& van Albada, T. S., 2007, MNRAS, 376, 1513

Noordermeer, E., \& Verheijen, M. a. W., 2007, MNRAS, 381, 1463

Noterdaeme, P., Petitjean, P., Ledoux, C., \& Srianand, R., 2009, A\&A, 505, 1087

Noterdaeme, P., Petitjean, P., Carithers, W. C., et al., 2012, A\&A, 547, L1

Obreschkow, D., Klöckner, H.-R., Heywood, I., Levrier, F., \& Rawlings, S., 2009, ApJ, 703, 1890

Obreschkow, D., Croton, D., De Lucia, G., Khochfar, S., \& Rawlings, S., 2009, ApJ, 698, 1467

Obreschkow, D., \& Rawlings, S. 2009, ApJ, 696, L129

Oosterloo, T., Fraternali, F., \& Sancisi, R., 2007, AJ, 134, 1019

Oosterloo, T., \& van Gorkom, J., 2005, A\&A, 437, L19

Ott, J., Stilp, A. M., Warren, S. R., et al., 2012, AJ, 144, 123

Panter, B., Jimenez, R., Heavens, A. F., and Charlot, S., 2007, MNRAS, 378, 1550

Pelupessy, F. I., Papadopoulos, P. P., \& van der Werf, P. 2006, ApJ, 645, 1024

Pelupessy, F. I., \& Papadopoulos, P. P. 2009, ApJ, 707, 954

Pidopryhora, Y., Lockman, F. J., and Shields, J. C., 2007, ApJ, 656, 928

Pisano, D. J., Barnes, D. G., Gibson, B. K., et al., 2007, ApJ662, 959

Popping, A., Meyer, M., Staveley-Smith, L., Obreschkow, D., Jozsa, G.I., Pisano, D.J. \& the HI Science Working Group, "Observations of the Intergalactic Medium and the Cosmic Web in the SKA era", in proc. Advancing Astrophysics with the Square Kilometre Array, PoS(AASKA14) 132

Popping, G., Somerville, R. S., \& Trager, S. C. 2014, MNRAS, 442, 2398

Popping, A., Davé, R., Braun, R., \& Oppenheimer, B. D., 2009, A\&A, 504, 15

Power, C., Baugh, C. M., \& Lacey, C. G. 2010, MNRAS, 406, 43

Prochaska, J. X., Herbert-Fort, S., \& Wolfe, A. M. 2005, ApJ, 635, 123

Putman, M. E., Peek, J. E. G., and Joung, M. R., 2012, ARA\&A, 50, 2012, 491

Rafieferantsoa, M., Davé, R., Anglés-Alcazar, D., et al. 2014, arXiv:1408.2531

Rahmati, A., \& Schaye, J. 2014, MNRAS, 438, 529 
Rao, S., \& Briggs, F., 1993, ApJ, 419, 515

Rao, S. M., Turnshek, D. A., \& Nestor, D. B. 2006, ApJ, 636, 610

Rhee, J., Zwaan, M. A., Briggs, F. H., et al. 2013, MNRAS, 435, 2693

Robertson, B. E., \& Kravtsov, A. V. 2008, ApJ, 680, 1083

Ryan-Weber, E. V., Begum, A., Oosterloo, T., et al., 2008, MNRAS, 384, 535

Sancisi, R., Fraternali, F., Oosterloo, T., \& van der Hulst, T., 2008, ARA\&A, 15, 189

Schiminovich, D., Catinella, B., Kauffmann, G., et al., 2010, MNRAS, 408, 919

Scott, T. C., Sengupta, C., Verdes Montenegro, L., et al., 2014, A\&A, 567, A56

Serra, P. 2011, Fornax, Virgo, Coma et al., Stellar Systems in High Density Environments, 49P

Serra, P., Oosterloo, T., Morganti, R., et al., 2012, MNRAS, 422, 1835

Springob, C. M., Haynes, M. P., \& Giovanelli, R. 2005, ApJ, 621, 215

Schönrich, R. and Binney, J., 2009, MNRAS, 396, 203

Shetty, R., \& Ostriker, E. C. 2012, ApJ, 754, 2

Sorce, J. G., Courtois, H. M., Tully, R. B. et al., 2013, ApJ, 765, 94

Swaters, R. A., Sancisi, R., van Albada, T. S., \& van der Hulst, J. M., 2009, A\&A, 493, 871

Tacconi, L. J., Neri, R., Genzel, R., et al. 2013, ApJ, 768, 74

Tully, R. B., \& Fisher, J. R., 1977, A\&A, 54, 661

Tully, R. B.. \& Courtois, H. M., 2012, ApJ, 749, 78

Tumlinson, J., Thom, C., Werk, J.K., Prochaska, J.X., Tripp, T.M., Katz, N. et al., 2013, ApJ, 777, 59

van der Hulst, T., \& Sancisi, R., 1988, AJ, 95, 1354

van der Hulst, J. M., van Albada, T. S., \& Sancisi, R., 2001, Gas and Galaxy Evolution, 240, 451

van der Hulst, J. M., \& Blok, W. J. G. d., The Cool ISM in Galaxies 2013, Planets, Stars and Stellar Systems. Volume 6: Extragalactic Astronomy and Cosmology, 183

Verdes-Montenegro, L., Yun, M. S., Williams, B. A., et al., 2001, A\&A, 377, 812

Verheijen, M. A. W., 2001, ApJ, 563, 694

Verheijen, M. A. W., \& Sancisi, R., 2001, A\&A, 370, 765

Verheijen, M. A. W., Galaxy evolution in dense environments: a concise HI perspective 2004, IAU Colloq. 195: Outskirts of Galaxy Clusters: Intense Life in the Suburbs, 394

Verheijen, M., van Gorkom, J. H., Szomoru, A., et al., 2007, ApJ, 668, L9

Verheijen, M., Oosterloo, T., Heald, G., \& van Cappellen, W., 2009, Panoramic Radio Astronomy: Wide-field 1-2 GHz Research on Galaxy Evolution

Verheijen, M. et al. 2014, 7th International PHISCC Workshop, The Challenges of the Upcoming H I Surveys

Wakker, B. P. and van Woerden, H., 1997, ARA\&A, 35, 217

Walter, F., Brinks, E., de Blok, W. J. G., et al., 2008, AJ, 136, 2563

Wang, J., Kauffmann, G., Józsa, G. I. G., et al. 2013, MNRAS, 433, 270

Westmeier, T., Braun, R., Thilker, D., 2005, A\&A, 436, 101

Wong, O. I., Webster, R. L., Kilborn, V. A., Waugh, M., \& Staveley-Smith, L., 2009, MNRAS, 399, 2264

Zafar, T., Péroux, C., Popping, A., et al. 2013, A\&A, 556, A141

Zaritsky, D., Courtois, H., Muñoz-Mateos, J.-C. et al., 2014, AJ, 147, 134

Zwaan, M. A., van der Hulst, J. M., de Blok, W. J. G., \& McGaugh, S.S., 1995, MNRAS, 273 L35 
Zwaan, M. A., Staveley-Smith, L., Koribalski, B. S., et al., 2003, AJ, 125, 2842

Zwaan, M. A., Meyer, M. J., Staveley-Smith, L., \& Webster, R. L., 2005, MNRAS, 359, L30

Zwaan, M. A., van der Hulst, J. M., Briggs, F. H., Verheijen, M. A. W., \& Ryan-Weber, E. V., 2005, MNRAS, 364, 1467 